\title{
Primary Genetic Investigation of a Hyperlipidemia Model: Molecular Characteristics and Variants of the Apolipoprotein E Gene in Mongolian Gerbil
}

\author{
Yuehuan Liu, ${ }^{1,2}$ Jiusheng Wu, ${ }^{1}$ Qiaojuan Shi, ${ }^{2}$ Honggang Guo, ${ }^{2}$ \\ Huazhong Ying, ${ }^{2}$ and Ningying $\mathrm{Xu}^{1}$ \\ ${ }^{1}$ College of Animal Sciences, Zhejiang University, Hangzhou 310058, China \\ ${ }^{2}$ Zhejiang Academy of Medical Sciences, Hangzhou 310013, China \\ Correspondence should be addressed to Ningying Xu; nyxu@zju.edu.cn
}

Received 22 January 2014; Revised 6 May 2014; Accepted 8 May 2014; Published 1 June 2014

Academic Editor: Qinghua Nie

Copyright (c) 2014 Yuehuan Liu et al. This is an open access article distributed under the Creative Commons Attribution License, which permits unrestricted use, distribution, and reproduction in any medium, provided the original work is properly cited.

The objective of this work was to establish a novel Mongolian gerbil (Meriones unguiculatus) hyperlipidemia model and to investigate its susceptibility genetic basis. Two rodent (gerbil and rat) hyperlipidemia models were induced by feeding a high fat/high-cholesterol (HF/HC) diet. There were significant increases of serum total cholesterol, triglycerides, low-density lipoprotein cholesterol (LDL-C), and high-density lipoprotein cholesterol (HDL-C) in gerbils within a 4-week modeling period. About 10-30\% of $>8$-month-old individuals developed hyperlipidemia spontaneously. The apolipoprotein E (ApoE) gene was cloned by merging a sequence of rapid amplification of cDNA ends (RACE) and nested polymerase chain reaction products. The results revealed an open reading frame of $948 \mathrm{bp}$, encoding a protein of 298 amino acids. The gene without a $5^{\prime}$-UTR region in the first intron was highly homologous to human Apo-A-I and rat Apo-A-IV. The distribution of expression of the ApoE gene in liver, brain, heart, lung, kidney, and adrenal gland was detected by an ABC immunohistochemical procedure. Three single nucleotide polymorphisms (SNPs; C97T, G781T, and A1774T) were first found using PCR-single-strand conformation polymorphism (PCR-SSCP) in a closed population containing 444 animals. Correlation analysis confirmed that new SNPs, age, and gender were associated significantly $(P<0.05)$ with hyperlipidemia.

\section{Introduction}

Current rodent models for hyperlipidemia include induced rats, guinea pigs, Syrian hamsters, and gene-knocked-out mice worldwide [1]. Hyperlipidemia is major risk factor for the development of cardiovascular disease. Despite the multifactorial pathogenesis of hyperlipidemia, high intakes of calories and fats (cholesterol-rich fats and saturated fatty acids) are widely considered as major contributing factors [2]. Although rats and mice have been extensively used in biomedical research to generate data on metabolic diseases, they have limitations as models of lipid metabolism in humans (for example, lipid metabolism particularly in changes of lipoproteins and serum cholesterol levels in response to dietary factors differ significantly between rats and humans) [3]. Thus, exploiting a new experimental animal model in studying hyperlipidemia promotes extrapolation of data since fatty acids and cholesterol play important roles in the etiology of diseases.

Early work with an induced hyperlipidemia model in rodents (rat, mouse, and gerbil) fed a high fat/highcholesterol (HF/HC) diet showed the Mongolian gerbils (Meriones unguiculatus) model could be induced within 1 week without adding lard, egg yolk powder, or drugs to restrain thyroid activity $[4,5]$. Feeding an $\mathrm{HF} / \mathrm{HC}$ diet (included 2\%(w/w) cholesterol and 7\% (w/w) lard) can induce a prompt increase of total cholesterol and LDL-C concentration in peripheral blood and liver, which remains stable within 4 weeks [6]. Feeding an $\mathrm{HF} / \mathrm{HC}$ diet also led to a significant increase in serum total cholesterol (TC), triglyceride (TG), low-density lipoprotein cholesterol (LDL-C), and high-density lipoprotein cholesterol (HDL-C) in gerbils, 
which were found in a time-dependent manner during $0 \sim 16$ weeks' feeding. Hepatic lipid vacuolization and even fibrosis in gerbils were greatly formed in response to the high fat diet with the characteristic of serum LDL-C increase, while those index remained unchanged in rats. Furthermore, serum lecithin cholesterol acyl transferase (LCAT) activities in the hyperlipidemia gerbils were significantly higher than those in the normal ones, which were also in line with increased LDLC-TG secretion rate and impaired hepatic function in gerbils in response to the $\mathrm{HF} / \mathrm{HC}$ diet. Therefore, gerbils were considered to be more sensitive to high fat diet and less timeconsuming in forming hyperlipidemia, with similar response in increased LDL-C levels to cholesterol as human (there are two distinct features in lipid content in this model compared to human. One is its LDL-based particle. Another is over 30\% free cholesterol in serum) [7]. Moreover, there are about 10$30 \%$ Z:ZCLA gerbils (a new experimental strain in China) of over-8-month-old gerbils (named old gerbils) prevalent for hyperlipidemia (hypercholesterolemia). But their genetic basis is unknown.

The human apolipoprotein E (ApoE) gene is located on 19q13.2, where it is linked with ApoC I, II, and IV. The $3.7 \mathrm{~kb}$ gene, which includes 4 exons and 3 introns, encodes a 34,200 Da glycoprotein composed of 299 amino acids [8]. As a major protein involved in lipoprotein metabolism and cardiovascular disease, ApoE might bidirectionally adjust action under the interaction of diet as environmental factors and genetic susceptibility, which has been detected in a variety of tissues, including liver, kidney, and brain [9]. It is well documented that the polymorphism and sequence variation of the ApoE gene have a strong correlation with lipid metabolism [10], cardiovascular diseases, cerebrovascular diseases, Alzheimer's disease, brain trauma, immune regulation, nerve regeneration and repair, and other forms of physiological activity $[11,12]$. Up to now, the role in many aspects on ApoE has come from human cell models [13] and ApoE gene-deficient mice [14]. The hyperlipidemia and atherosclerosis animal pathogenesis and how ApoE affects hyperlipidemia and atherosclerosis are not entirely clear.

There has been no report of the relationship between the ApoE gene and cholesterol metabolism in Mongolian gerbils. In order to identify the genetic basis (discovered major genes or functional SNPs) and sequence structure and their effects on cholesterol metabolism, a total of 534 gerbils (model group and breeding population) were used to investigate the characteristics of the entire ApoE gene sequence, the polymorphism, and its relationship with the hyperlipidemia phenotype.

\section{Materials and Methods}

2.1. Animals and Diets. Sixty male gerbils (Meriones unguiculatus) of 50-70 g body weight, 60 male Sprague Dawley rats of $180-200 \mathrm{~g}$ body weight, and $30>8$ months old gerbils, referred to here as old gerbils (manifested hyperlipidemia spontaneously), of 110-150 g body weight were obtained from the Zhejiang Center of Laboratory Animal, China. Five animals were housed together in a standard cage and kept at a temperature of $20-26^{\circ} \mathrm{C}$ with a $12 \mathrm{~h}$ light $/ 12 \mathrm{~h}$ dark photoperiod. All experiments were done in accord with local guidelines and were approved by the Ethics Committee for Research on Laboratory Animal Use of Zhejiang Academy of Medicine Sciences. All animals (rats and gerbils) were given access $a d$ lib to a commercially available standard diet (Zhejiang Center of Laboratory Animals, China) for 1 week before the experiment was started. The standard diet was produced in accordance with GB14924-2010 standard. It is composed of water and other volatile substances $\leqslant 10 \%$, crude protein $\geqslant 18 \%, \geqslant 4 \%$ crude fat, crude fiber $\leqslant 5 \%$, crude ash $\leqslant 8 \%$, calcium $10-18 \mathrm{~g}$, total phosphorus $6-12 \mathrm{~g}$, calcium and phosphorus ratio 1.2-1.7, gerbils nutrition standards 4th edition (National Academy Press, Washington, D.C. 1995; $2-5 \%$ crude fat, crude protein $16-25 \%$, calcium $5.0 \mathrm{~g}$, and phosphorus $3.0 \mathrm{~g}$ ). From day zero of the experiment, control animals ( $n=30$, ZC group) and the old gerbils group $(n=30$, LN group) were fed the standard diet, and all other rats and gerbils ( $n=30$, GZ group) were fed an HF/HC diet composed of $80.5 \%(\mathrm{w} / \mathrm{w})$ standard diet, $2 \%(\mathrm{w} / \mathrm{w})$ cholesterol, $7 \%(\mathrm{w} / \mathrm{w})$ lard, $10 \%(\mathrm{w} / \mathrm{w})$ yolk powder, and $0.5 \%(\mathrm{w} / \mathrm{w})$ bile salts [7].

\subsection{Modeling, Sampling, Biochemical Testing, and Statistical} Analysis. The influence of the different diets was observed during 4 weeks. During this time, we recorded body weight. After withholding food for $12 \mathrm{~h}$, all animals were sacrificed by anesthesia with carbon dioxide; whole blood samples were collected for biochemical analysis and two small pieces of each of liver, brain, heart, spleen, lung, kidney, and adrenal gland were collected. One piece of each tissue was kept at $-80^{\circ} \mathrm{C}$ for gene cloning and the other piece was used for routine histology tests (staining with Oil Red O and H\&E) and ApoE immunohistochemical analysis. Serum was extracted for the detection of triglycerides (TG), total cholesterol (TC or $\mathrm{CHO}$ ), high-density lipoprotein cholesterol (HDL-C), and low-density lipoprotein cholesterol (LDL-C).

\subsection{To Acquire the Whole Sequence of the ApoE Gene Encod-} ing Area. DNA was extracted with a tissue genomic DNA extraction kit (Shanghai Invitrogen, China). Total RNA were extracted with TRIzol reagent (Shanghai Invitrogen, China) and quantified by spectrophotometry. First strand cDNA was synthesized using a reverse transcriptase kit (Promega) and the middle region of the gene was cloned with primers APOF1/APO-R1 and APO-F2/APO-R2. Rapid amplification of cDNA ends (RACE) was used to obtain the $5^{\prime}$ and $3^{\prime}$-flanking regions with the FirstChoice RLM-RACE Kit (Ambion). An additional four pairs of nested primers (APO-intron-F1APO-intron-R8) were designed to amplify the introns. All amplified products were sequenced and constructed to one contig. A primer pair termed ApoE-full was designed to validate the contig and used to screen the SNP in the gerbil experimental population; 5 overlapping primer pairs were designed for PCR-SSCP (PCR-single-strand conformation polymorphism). All primers are given in Table 1 . The PCR protocol was as follows: all reactions were done in $25 \mu \mathrm{L}$ volumes containing $0.25 \mu \mathrm{L}$ of LATaq DNA polymerase. The reaction sequence was $94^{\circ} \mathrm{C}$ for $4 \mathrm{~min}$, then 35 cycles at $94^{\circ} \mathrm{C}$ for $30 \mathrm{~s}, 55-58^{\circ} \mathrm{C}$ for $30 \mathrm{~s}, 72^{\circ} \mathrm{C}$ for $45 \mathrm{~s}$, followed by 
TABLE 1: Primers listed in this paper.

\begin{tabular}{|c|c|c|}
\hline Primer name & Primer sequence & Size (bp) and location \\
\hline APO-F1 & $5^{\prime}$ CCSTGCTGTTGGTCMCATT $3^{\prime}$ & Degenerate primers (nest primer) \\
\hline APO-R1 & $5^{\prime}$ GAGGCCTGTATCTTCTCC $3^{\prime}$ & $600 \mathrm{bp}$ \\
\hline APO-F2 & $5^{\prime}$ GAGCYGGAGGTGACAGAT $3^{\prime}$ & \\
\hline APO-R2 & $5^{\prime}$ GGCGMTGCATGTCTTCCACTA $3^{\prime}$ & \\
\hline APO-E-R1 & $5^{\prime}$ GCT CCT TTG TGT AAG CCT TCA C $3^{\prime}$ & $5^{\prime}$ RACEprimer (nest primer) \\
\hline APO-E-R2 & $5^{\prime}$ ATG GTG TCC TCT ATC AGA ACC GTC $3^{\prime}$ & $400 \mathrm{bp}$ \\
\hline APO-E-F1 & $5^{\prime}$ CAA GAT GGA GGA GCA GAC ACA $3^{\prime}$ & $3^{\prime}$ RACEprimer (nest primer) \\
\hline APO-E-F2 & $5^{\prime}$ GGA GGA GCA GAC ACA GCA GAT $3^{\prime}$ & $300 \mathrm{bp}$ \\
\hline APO-intron-F1 & $5^{\prime}$ ATGAAGGCTCTGTGGGCTG $3^{\prime}$ & Fish for ApoE introns \\
\hline APO-intron-R1 & $5^{\prime}$ AAGCCTTCACTTCTGTCATGGTGT $3^{\prime}$ & (nest primer) $195 \mathrm{bp}$ \\
\hline APO-intron-F2 & $5^{\prime}$ GTCGCGTTGTTGGCAGGAT $3^{\prime}$ & \\
\hline APO-intron-R2 & $5^{\prime}$ GTCCTCTATCAGAACCGTCAG $3^{\prime}$ & \\
\hline APO-intron-F3 & $5^{\prime}$ TGCAGACGCTGTCTGACCA $3^{\prime}$ & Fish for ApoE introns \\
\hline APO-intron-R3 & $5^{\prime}$ CCAGCATGGTCTGTACCTC $3^{\prime}$ & (nest primer) $169 \mathrm{bp}$ \\
\hline APO-intron-F4 & $5^{\prime}$ GACGGTTCTGATAGAGGACAC $3^{\prime}$ & \\
\hline APO-intron-R4 & $5^{\prime}$ GCCCAGTCTGTTGCGAAG $3^{\prime}$ & \\
\hline APO-intron-F5 & $5^{\prime}$ GACATGGAAGACCTTCGCAACA $3^{\prime}$ & Fish for ApoE introns \\
\hline APO-intron-R5 & $5^{\prime}$ CCTGGTTGCCCATCAGCT $3^{\prime}$ & (nest primer) $287 \mathrm{bp}$ \\
\hline APO-intron-F6 & $5^{\prime}$ GCAGCGAGGTACAGACCA $3^{\prime}$ & \\
\hline APO-intron-R6 & $5^{\prime}$ GATGCGGGCACCCAAAGC $3^{\prime}$ & \\
\hline APO-intron-F7 & $5^{\prime}$ TGGTGGAGGAAGGTCGCC 3' & Fish for ApoE introns \\
\hline APO-intron-R7 & $5^{\prime}$ GTAGGGAGGATGGGATTGGT $3^{\prime}$ & (nest primer) $227 \mathrm{bp}$ \\
\hline APO-intron-F8 & $5^{\prime}$ GTCGGCTGGAGCTGATGG $3^{\prime}$ & \\
\hline APO-intron-R8 & $5^{\prime}$ GGGATTGGTAGCCACGGA $3^{\prime}$ & \\
\hline APO-Full-F1 & $5^{\prime}$ CCC GAA GGC TAA GGT TTT G $3^{\prime}$ & Contig primer \\
\hline APO-Full-R1 & $5^{\prime}$ ACC TGC TGG TCG TGG AT $3^{\prime}$ & (Nest primer) $1800 \mathrm{bp}$ \\
\hline APO-Full-F2 & $5^{\prime}$ GGC TGG TTC ATC ACA GTT GTG $3^{\prime}$ & \\
\hline APO-Full-R2 & $5^{\prime}$ TGG AGA GGG ATC TTC ATT GAC TCT $3^{\prime}$ & \\
\hline liuF1 & 5' GGTCGCGTTGTTGGCAGGTA 3' & SSCP primer, $410 \mathrm{bp}$ \\
\hline liuR1 & $5^{\prime}$ ATAGGACAGAGCTGAGACCA $3^{\prime}$ & \\
\hline liuF2 & $5^{\prime}$ AGACGCTGTCTGACCAGGTC $3^{\prime}$ & SSCP primer, $449 \mathrm{bp}$ \\
\hline liuR2 & $5^{\prime}$ AGGGCCTAGACAGAAGGGCC $3^{\prime}$ & \\
\hline liuF3 & $5^{\prime}$ CTGGAGCTGATGGGCAACCA $3^{\prime}$ & SSCP primer, $275 \mathrm{bp}$ \\
\hline liuR3 & $5^{\prime}$ GGGGTGGAGAGGGATCTTCA $3^{\prime}$ & \\
\hline liuF4 & $5^{\prime}$ CCCCCGAAGGCTAAGGTTTT $3^{\prime}$ & SSCP primer, $225 \mathrm{bp}$ \\
\hline liuR4 & $5^{\prime}$ ATAAAAAAGCTGCTCGGGGC $3^{\prime}$ & \\
\hline liuF5 & $5^{\prime}$ CTGGAGCTGAT GGGCAACCA $3^{\prime}$ & SSCP primer, $347 \mathrm{bp}$ \\
\hline liuR5 & $5^{\prime}$ AAGTAAGGGCCACCAGAGGG 3' & \\
\hline
\end{tabular}

*All primers were synthesized by Shanghai Sangon, China.

$72^{\circ} \mathrm{C}$ for 10 min and then $10^{\circ} \mathrm{C}$ insulation (Mastercycler Pros, Eppendorf).

Nine reference sequences from GenBank (human, M10065 and af261279; mouse, d00466; rat, J02582; chimpanzee, af200499; cattle, DQ538523; gorilla, AF200502; pig, u70240; and gibbon, AF200508) were used to calculate the phylogenetic tree with DNAstar software. Sequences of human (NC_000019), mouse (NC_000073), rat (J02582), tree shrew (AF303830), and rabbit (NM_001082643) from GenBank were used to compare the mRNA structure.
The structure was compared with a RNA structure software (version 5.1).

2.4. Immunohistochemical Analysis. Tissue samples collected from gerbil groups ZC, GZ, and LN were used for immunohistochemical analysis of ApoE using the SABC kit (Booster Bioengineering Institute, Wuhan, China). Positive cells showed yellow or brown particles or clumps in the cytoplasm with a blue-stained nucleus, photographed with a $200 \mathrm{X}$ magnification objective lens. 
TABLE 2: The blood biochemical index of SD rat and Mongolian gerbil.

\begin{tabular}{lccccc}
\hline & GZ rat & ZC rat & GZ gerbil & ZC gerbil & LN gerbil \\
\hline CHO $(\mathrm{mg} / \mathrm{dL})$ & $255.29 \pm 105.96^{* *}$ & $103.66 \pm 27.46$ & $505.93 \pm 266.51^{* *}$ & $116.04 \pm 33.65$ & $230.92 \pm 144.28^{*}$ \\
TG $(\mathrm{mg} / \mathrm{dL})$ & $89.64 \pm 15.98$ & $120.70 \pm 55.91$ & $87.86 \pm 76.33$ & $78.10 \pm 31.95$ & $1403.40 \pm 959.9^{* *}$ \\
HDL $(\mathrm{mg} / \mathrm{dL})$ & $38.25 \pm 7.73$ & $24.34 \pm 6.96$ & $751.55 \pm 386.17^{* *}$ & $72.64 \pm 17.77$ & $166.15 \pm 55.77$ \\
LDL $(\mathrm{mg} / \mathrm{dL})$ & $75.87 \pm 22.06$ & $81.68 \pm 20.13$ & $379.75 \pm 201.94^{* *}$ & $96.78 \pm 17.03$ & $96.78 \pm 73.55$ \\
\hline
\end{tabular}

${ }^{*}$ Two-tailed Student's $t$-test was applied to calculate difference between treatments, ${ }^{* *}$ means $P<0.01$, and ${ }^{*}$ means $P<0.05$.

2.5. Polymorphism Detection, Allele Frequency, and Association Analysis. DNA samples from the gerbil ZC, GZ, and LN groups were used to verify the gene contig and screen SNP. Polymerase chain reaction-single-strand conformation polymorphism (PCR-SSCP) was used to analyze DNA samples from a total of 444 animals; these animals were further distributed into several subgroups according to generation $(45,46,47,49)$, age period ( $149<8$ months old, $829-17$ months old, 46 18-26 months old), and gender (o, ơ). The PCR products were separated by PAGE, stained with silver, and photographed with a gel imaging system (Bio-Rad).

2.6. Correlation Analysis of ApoE Gene SNP with Hyperlipidemia of Gerbils. All data were analyzed by GLM procedure of SPSS16.0 software and presented as Mean \pm SD. Correlation analysis was used to analyze the difference between SNP genotype distributions among groups. Tukey's multiple comparison was conducted to detect the difference among means. Biochemical indexes were compared by the 2-tailed Student's $t$-test. The statistically significant difference was set at $P<0.05$. Genotypes and allele frequencies of three SNPs were computed by EXCEL software, and a Chi-square test was used to detect the significant difference.

The model used to analyze the correlation between the individual's genotype and hyperlipidemia trait was as follows:

$$
Y_{i j k l m}=\mu+S_{i}+F_{j}+G_{k}+l_{l}+P m+\beta * X_{i j k l m}+e_{i j k l m},
$$

where $Y_{i j k l m}$ is trait phenotypic value, $\mu$ is group average, $S_{i}$ is sex effect, $E_{j}$ is environment effect, $G_{k}$ is genotype effect, $l_{l}$ is generation effect, $P_{m}$ is age effect, $\beta$ is regression coefficient for hyperlipidemia trait, $X_{i j k l m}$ is hyperlipidemia indicator as covariate, and $e_{i j k l m}$ is residuals value.

\section{Results}

3.1. Biochemical Index and Pathology of Gerbil Hyperlipidemia Model. The serum level of total cholesterol (TC or CHO), triglycerides (TG), LDL-C, and HDL-C is given in Table 2. The serum TC level of the gerbil GZ group multiplied rapidly to 10 -fold higher compared to the gerbil $\mathrm{ZC}$ group and sustained a 5 -fold increase over 4 weeks $(P<0.01)$ but the increase was only 2 -fold for the gerbil LN group. LDL-C and HDL-C of the gerbil GZ group increased rapidly to 4-fold greater compared to the gerbil ZC group and remained stable over four weeks. By contrast, the gerbil ZC group and rats were basically unchanged. The increased level of cholesterol was due mainly to elevated LDL-C rather than HDL-C. The $\mathrm{HDL}-\mathrm{C} / \mathrm{TC}$ ratio of the gerbil GZ group was lower compared to the gerbil ZC and rat groups. It is easy to conclude that the serum cholesterol transport in the gerbil GZ group depended largely on LDL-C, which is analogous to human.

The GZ group gerbils were heavier compared to the ZC group. Body fat deposition, fatty liver, and swollen spleen were observed in GZ group gerbils. Staining with Oil Red $\mathrm{O}$ and $\mathrm{H} \& \mathrm{E}$ suggested that after 4 weeks, gerbil liver tissue exhibited significant fatty degeneration of liver cells, which was seen also in LN group gerbils (Figure 1). Liver lobules of ZC group gerbils and rats were evident; no lipid deposition was seen by staining hepatic cells.

3.2. Molecular Cloning and Sequence Analysis of the ApoE Gene in the Mongolian Gerbil. The cDNA sequence (CDS) of the gerbil ApoE gene is $1135 \mathrm{bp}$ long. The full-length gene is composed of a $5^{\prime}$-UTR (65 bp), a $3^{\prime}$-UTR (119bp), 3 exons ( $44 \mathrm{bp}, 181 \mathrm{bp}$, and $726 \mathrm{bp}$ ), and 2 introns (521 bp and $421 \mathrm{bp}$ ), which were constructed to a $2077 \mathrm{bp}$ contig (accession number EU834053). Figure 2 shows the structure of the complete gene. The typical start codon (ATG) and a stop codon (TGA) were located on the ApoE gene sequence but the exon/intron boundaries were in conflict with the GT/AG rule. The poly-(A) signal site (AATAAA) was located $30 \mathrm{bp}$ upstream from the poly-(A) tail. Four-copy ATTTA motifs, which are known as the most important determinants of RNA stability, were located at 2 intron sequences. The gerbil ApoE gene sequences were registered in GenBank (accession number EU834053). Clustering analysis indicated gerbil, mouse, and rat are combined into a separate group. The homology of ApoE CDS of gerbils with mouse and rat reached $86 \%, 79.2 \%$ with human and $<78 \%$ with nonhuman primates or domestic animals. The Mongolian gerbil ApoE mRNA open reading frame (ORF) was $951 \mathrm{bp}$, the free energy was $-31.0 \mathrm{kcal}$, the stem bases were $552 \mathrm{bp}$, and the ring bases were $501 \mathrm{bp}$; the human ORF was $954 \mathrm{bp}$, the free energy was $-625.80 \mathrm{kcal}$, the stem bases were $536 \mathrm{bp}$, and the ring bases were $610 \mathrm{bp}$.

A gene that coded a protein of 316 amino acids was predicted, which included an 18-amino-acid signal peptide. The sequence contains 50 negatively charged residues (Asp + Glu) and 47 positively charged residues, which form $3 \alpha$ helical regions and a signal peptide. The LDL-R binding site (LRKMRKRLLR) was found in positions 137-146 of the mature protein. The detailed sequence of the mature protein was registered in GenBank (accession number EU834053).

3.3. Distribution of ApoE Protein in the Mongolian Gerbil . Immunohistochemical staining showed ApoE-positive reactions in the liver, brain, heart, lung, kidney, and adrenal 


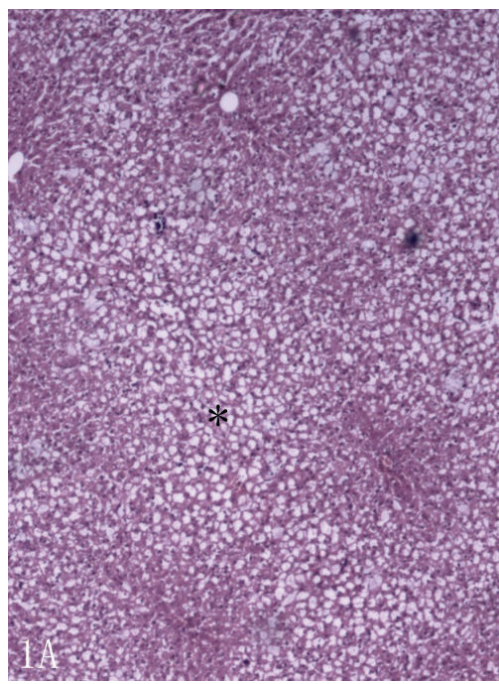

(a)

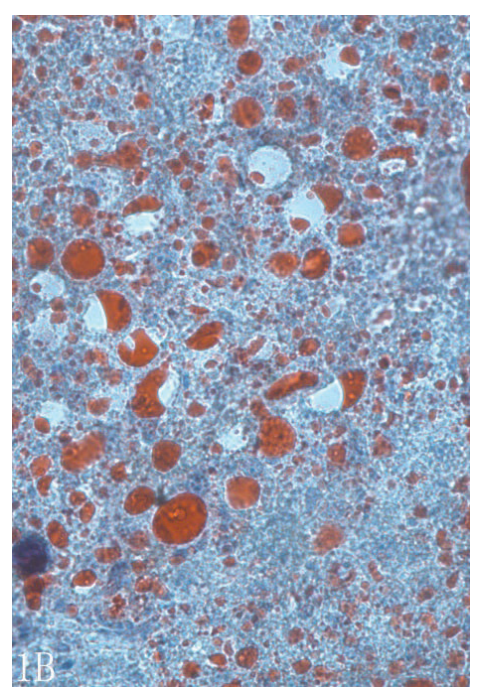

(b)

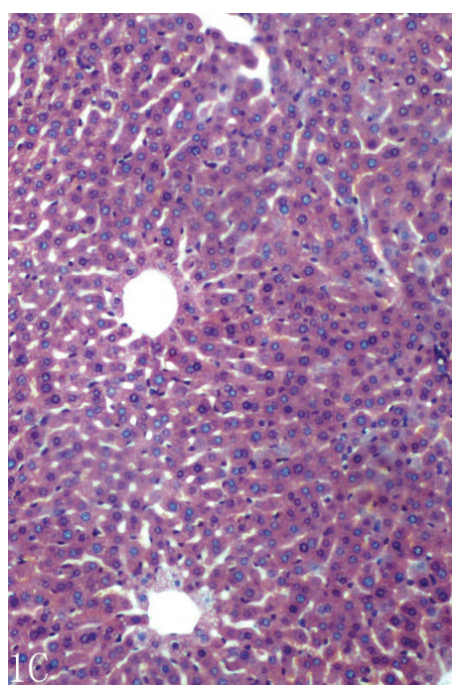

(c)

FIGURE 1: (a) Pathological section (HE staining) from Mongolian gerbil hyperlipidemia (Olympus, 10X objective lens); (b) pathological section (oil red staining) from Mongolian gerbil hyperlipidemia (Olympus, 40X objective lens); (c) pathological section (HE staining) from controlled group Mongolian gerbil (Olympus, 40X objective lens).

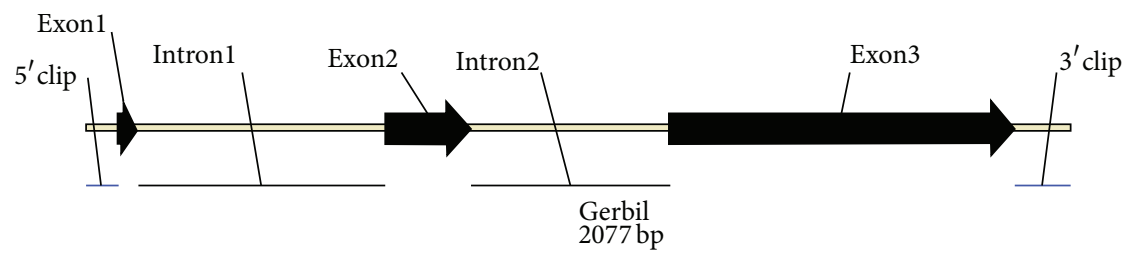

FIGURE 2: The genomic structure and sequences of ORF of the gerbil ApoE gene. The box structure, the gene sequence, includes the $5^{\prime}$-UTR (65 bp), three exons ( $44 \mathrm{bp}, 181 \mathrm{bp}$, and $726 \mathrm{bp}$, resp.), two introns (521 bp and $421 \mathrm{bp}$, resp.), and $3^{\prime}$-UTR (119 bp).

gland of the gerbil. In the brain, ApoE-positive products were distributed in both white and gray matter and staining was located mainly on the astrocytes. In the liver, all hepatocytes contained weakly immunoreactive granules. In the lung, ApoE-positive products were stained mainly in some pneumocytes lining the alveolar septa, especially in some type II pneumocytes. In the kidney, ApoE was located mainly on the epithelium in most of the proximal convoluted tubules. In the adrenal cortex, cells containing abundant granular reaction products were distributed mainly in the zona fascicularis and zona reticularis. In the zona glomerulosa, only the plasma membrane was stained. Expression of ApoE was observed in brain and kidney of the gerbil GZ and LN groups but the reaction was feeble and sparse in the $\mathrm{ZC}$ group. These results suggested that a variety of tissues and cells in the gerbil can synthesize and secrete ApoE (Figure 3).

3.4. SNP Screening and Analysis of ApoE Gene Polymorphism in the Mongolian Gerbil. Sequencing results of SNP screening and PCR-SSCP indicated that base mutations were detected by primer pairs 1,2 , and 5 . The polymorphic sites were located at positions $97(\mathrm{C} \rightarrow \mathrm{T}), 781(\mathrm{G} \rightarrow \mathrm{T})$ (Figure 4$)$, and 1774 $(\mathrm{A} \rightarrow \mathrm{T})$, which resulted in three changes of the amino acid sequence: at site 11 (signal peptide) Ala was mutated to Val, at site 65 leu was replaced to Leu, and at site 256 Lys was mutated to Met. Primer pair 1 distinguished 3 genotypes (CT, CC, and TT), primer pair 2 detected 3 genotypes (GG, GT, and TT), and primer pair 5 detected 2 genotypes (AT and $\mathrm{AA})$ in the test group. The genotype and gene frequencies are given in Table 3. At site 97, the frequency of allele $\mathrm{C}$ reached $>0.95$ and was the dominant position among generations. At sites $781(\mathrm{G} \rightarrow \mathrm{T})$ and $1774(\mathrm{~A} \rightarrow \mathrm{T})$, mutation genotype was relatively equilibratory, owing mainly to the possession of a considerable number of heterozygotes; the 781 heterozygotes accounted for the majority.

3.5. Association Analysis of the ApoE Gene by PCR-SSCP Polymorphism with Hyperlipidemia. The mutation effects in three sites were analyzed as follows (Table 4). In the SNPC97T site, 3 genotypes were correlated with body weight, $\mathrm{CHO}$, and TG $(P<0.01)$. The SNPG782T was correlated with body weight, $\mathrm{CHO}, \mathrm{HDL}, \mathrm{LDL}$, and TG $(P<0.01)$. The SNPA1774T site led to a TG difference. The ApoE gene was associated significantly with age $(P<0.01)$. Additionally, gender difference can display a change in serum levels of lipids $(P<0.05)$. In males, body weight, $\mathrm{CHO}, \mathrm{HDL}$, and TG were significantly higher $(P<0.05)$ compared to females. 


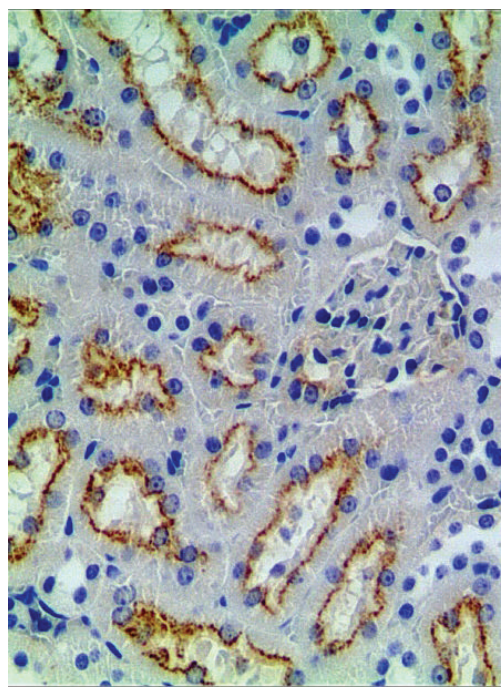

(a) $\mathrm{ZC}$ group $(\times 40$ objective lens $)$

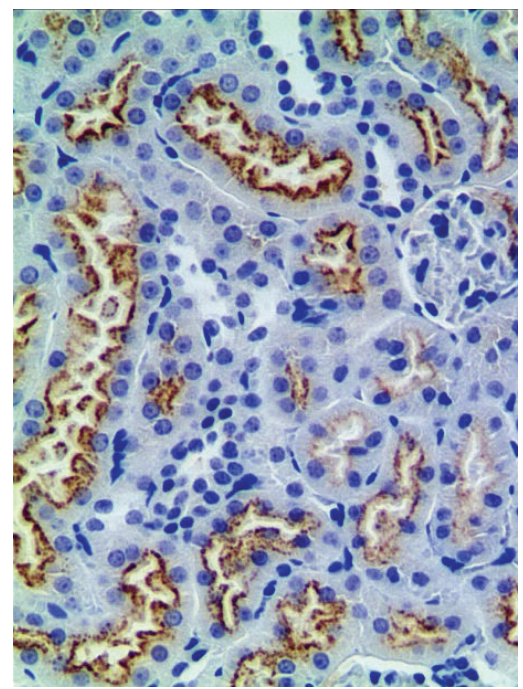

(b) GZ group ( $\times 40$ objective lens)

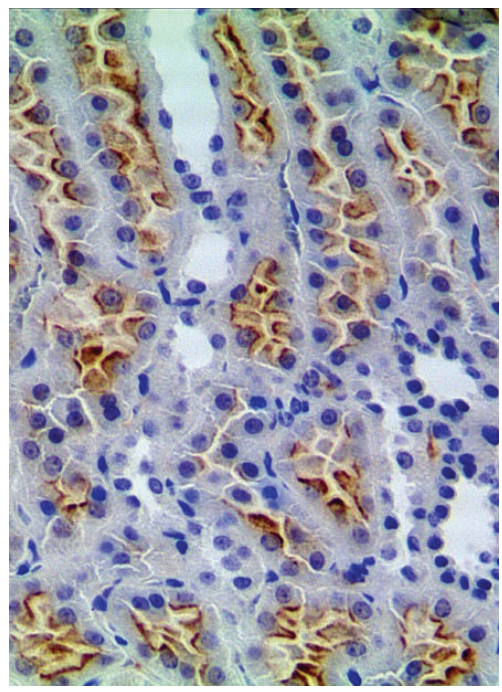

(c) $\mathrm{LN}$ group $(\times 40$ objective lens $)$

FIGURE 3: Presented the immunostaining results of kidney in each group. The positive products were distributed in the surface of the proximal convoluted tubules in the GZ, LN and ZC group.

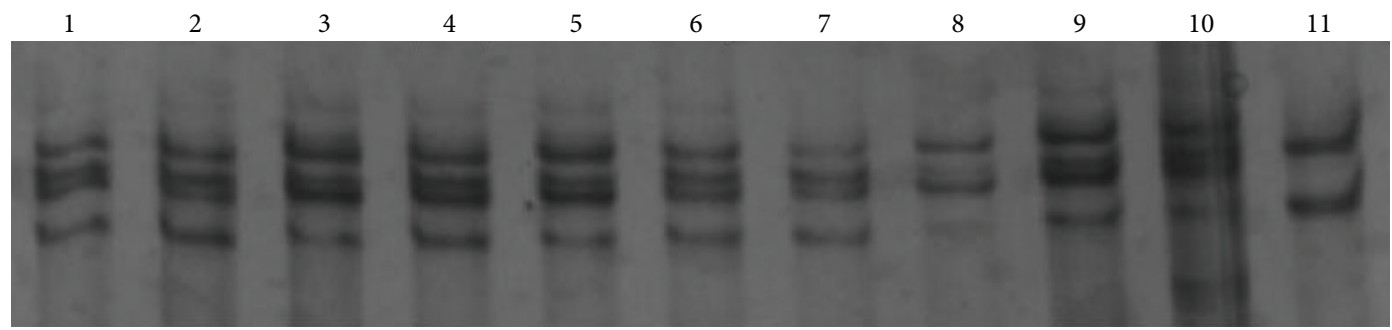

FIGURE 4: Genotypes of G781T SNP analyzed by PCR-SSCP, genotype: 1,9GT; 8TT; 11GG.

TABLE 3: Genotypes and allele frequencies of three SNPs in ZCLA Mongolia gerbil population.

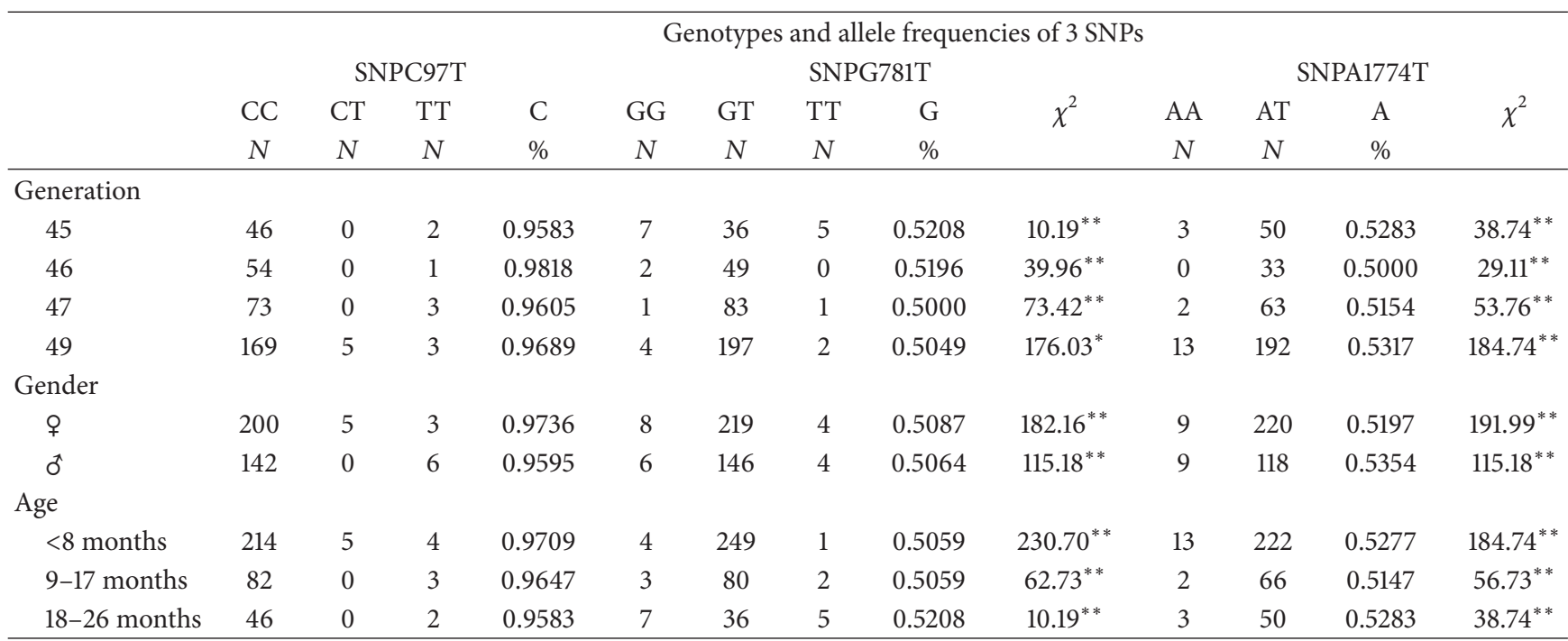

${ }^{*} \chi^{2}$ Test for Hardy-Weinberg equilibrium, $P<0.05,{ }^{* *} P<0.01$. 
TABLE 4: Association analysis between SNP genotypes and recorded traits

\begin{tabular}{|c|c|c|c|c|c|}
\hline & $\begin{array}{c}\text { Body weight } \\
X \pm \mathrm{SD}\end{array}$ & $\begin{array}{c}\mathrm{CHO} \\
X \pm \mathrm{SD} \\
\end{array}$ & $\begin{array}{c}\mathrm{HDL} \\
X \pm \mathrm{SD} \\
\end{array}$ & $\begin{array}{c}\mathrm{LDL} \\
X \pm \mathrm{SD} \\
\end{array}$ & $\begin{array}{c}\text { TG } \\
X \pm S D \\
\end{array}$ \\
\hline \multicolumn{6}{|l|}{ Gender } \\
\hline q & $76.2 \pm 21.9^{B}$ & $104.8 \pm 69.2^{\mathrm{b}}$ & $31.2 \pm 23.5^{\mathrm{b}}$ & $50.3 \pm 42.6$ & $648.5 \pm 491.3^{\mathrm{b}}$ \\
\hline o & $103.9 \pm 25.0^{\mathrm{A}}$ & $129.6 \pm 78.5^{\mathrm{a}}$ & $36.2 \pm 34.6^{\mathrm{a}}$ & $46.5 \pm 31.0$ & $758.3 \pm 527.7^{\mathrm{a}}$ \\
\hline \multicolumn{6}{|l|}{ Age } \\
\hline$<8$ months & $77.5 \pm 23.8^{\text {B }}$ & $92.1 \pm 35.6^{c}$ & $28.1 \pm 6.9^{\mathrm{B}}$ & $46.5 \pm 38.7^{\mathrm{B}}$ & $491.2 \pm 263.8^{B}$ \\
\hline 9-17 months & $106.8 \pm 22.3^{\mathrm{A}}$ & $150.1 \pm 98.2^{\mathrm{b}}$ & $41.9 \pm 49.6^{\mathrm{A}}$ & $46.5 \pm 31.0^{\mathrm{B}}$ & $1046.8 \pm 595.2^{A}$ \\
\hline $18-26$ months & $104.8 \pm 20.9^{\mathrm{A}}$ & $173.3 \pm 114.1^{\mathrm{a}}$ & $46.2 \pm 41.5^{\mathrm{A}}$ & $65.8 \pm 38.7^{\mathrm{A}}$ & $1172.4 \pm 680.4^{\mathrm{A}}$ \\
\hline \multicolumn{6}{|l|}{ C97T } \\
\hline $\mathrm{CC}$ & $88.0 \pm 26.7^{\mathrm{B}}$ & $114.5 \pm 73.5^{\mathrm{B}}$ & $33.5 \pm 30.8$ & $46.5 \pm 27.1$ & $705.1 \pm 490.7^{b}$ \\
\hline $\mathrm{CT}$ & $62.7 \pm 5.5^{\mathrm{C}}$ & $75.0 \pm 15.9^{\mathrm{B}}$ & $24.2 \pm 1.5$ & $31.0 \pm 7.7$ & $245.5 \pm 210.3^{c}$ \\
\hline $\mathrm{TT}$ & $110.1 \pm 32.3^{\mathrm{A}}$ & $167.9 \pm 104.0^{\mathrm{A}}$ & $38.5 \pm 16.9$ & $61.9 \pm 34.8$ & $1001.1 \pm 732.6^{\mathrm{a}}$ \\
\hline \multicolumn{6}{|l|}{ G781T } \\
\hline GG & $113.3 \pm 33.2^{\mathrm{A}}$ & $200.4 \pm 138.1^{\mathrm{A}}$ & $54.2 \pm 58.1^{\mathrm{A}}$ & $73.5 \pm 42.6^{\mathrm{A}}$ & $1025.5 \pm 714.8^{\mathrm{A}}$ \\
\hline GT & $86.4 \pm 26.0^{\mathrm{B}}$ & $109.5 \pm 68.5^{\mathrm{B}}$ & $31.9 \pm 23.5^{\mathrm{B}}$ & $46.5 \pm 38.7^{\mathrm{B}}$ & $669.1 \pm 487.5^{\mathrm{B}}$ \\
\hline $\mathrm{TT}$ & $106.7 \pm 22.7^{\mathrm{A}}$ & $163.2 \pm 106.4^{\mathrm{A}}$ & $70.8 \pm 108.8^{\mathrm{A}}$ & $58.1 \pm 34.8^{\mathrm{A}}$ & $1136.0 \pm 651.6^{\mathrm{A}}$ \\
\hline \multicolumn{6}{|l|}{ A1774T } \\
\hline AA & $84.9 \pm 22.7$ & $106.4 \pm 54.2$ & $39.6 \pm 49.2$ & $46.5 \pm 46.5$ & $486.5 \pm 359.4^{\mathrm{a}}$ \\
\hline AT & $85.6 \pm 26.2$ & $115.7 \pm 78.9$ & $33.5 \pm 30.0$ & $50.3 \pm 42.6$ & $701.0 \pm 529.4^{\mathrm{b}}$ \\
\hline
\end{tabular}

${ }^{*}$ Estimated value is the least squares mean \pm SD. Different lowercase letters $a, b$, and c indicate significant difference at 0.05 level; uppercase letters A, B, and C indicate significant difference at 0.01 level.

\section{Discussion}

Atherosclerosis or fatty liver disease is caused mainly by hyperlipidemia in animal models. An HF/HC diet is often used to induce a fatty liver disease model in Sprague Dawley or Wistar rats for evaluation of drug and lipid metabolism $[15,16]$. However, these rat models have some limitations in pathological practice. Recently, several expensive genetic animal models (such as ApoE gene knock-out mice or LDL$\mathrm{R}$ gene-deficient mice) were used for human hyperlipidemia and atherosclerosis research. In the gerbil model, cholesterol was added to the HF/HC diet to a final concentration of $2 \%(w / w)$ (about 10 -fold greater compared to endogenous amounts) [1]. The plasma content of total cholesterol (CHO) and LDL-C increased to $>500 \mathrm{mg} / \mathrm{dL}$ and $>300 \mathrm{mg} / \mathrm{dL}$, respectively, which is similar to the plasma level detected in LDL-R gene-deficient mice [17]. A short modeling period and its similarity to human hyperlipidemia led to the gerbil being the preferred model in pharmacology experiments $[7,18]$. Hyperlipidemia might occur spontaneously in the gerbil under certain nutritional conditions (refer to GB149242010 Chinese National Standards), which include many types of cholesterol and different fatty acids. It is likely that the expression of ApoE gene constantly regulated the uptake rate of LDL-C and sterol by the liver, the degradation of the rate-limiting enzymes for cholesterol, biosynthesis of the LDL-C receptor and the volume of cholesterol esters. When the levels of exogenous and endogenous cholesterol are decreased, it is necessary to increase circulating levels of LDLcholesterol to maintain steroidal homeostasis [19]. There are differences between the disorders of lipid metabolism in the rat and mouse models induced by the HF/HC diet. Two different clearance pathways (LDL-R/ApoB-100 and chylomicrons particulate/ApoB-48) can modify the ability to deal with the level of dietary cholesterol intake [20,21]. Because the gerbil has perfect ability in cholesterol intake [22], it is likely that there exists an LDL-R and ApoB-100 clearance pathway. It is unclear whether ApoB-48 and/or chylomicrons particulate pathways exist in the gerbil.

The gerbil ApoE gene contains 3 exons and 2 introns encoding a protein of 298 amino acids. The ApoE gene has 4 exons separated by 3 introns encoding a protein containing a signal peptide in human, pig, rat, mouse, and dog [2327]. The gorilla ApoE gene has 5 exons and 4 introns [28]. Quantitative sequence comparisons show that Apo-A-I, -A$\mathrm{IV}$, and $\mathrm{E}$ have evolved from a common ancestral gene [29]. The human Apo-A-I and Apo-A-IV genes in rat and mouse consist of 3 exons and 2 introns, and the first exon encodes a $5^{\prime}$-UTR and most of the signal peptide. Thus, the Apo-A gene has undergone multiple deletions that might affect its physiologic function, which is in contrast to all other known apolipoprotein genes [30,31]. This work showed the gerbil ApoE gene is similar to Apo-A gene in rat or mouse.

Comparison of the mRNA primary structure of ApoE in several species shows the ORF of the gerbil gene is only 1 amino acid shorter compared to human. The first amino acid that combined with the LDLR, which is critical for gene transcription and expression, is glutamine in gerbil and human [32]. A single cysteine residue is found within the signal peptide region of the rodent but there is no cysteine residue in the mature coding region. Met was found instead of Leu in the gerbil, as in the rat, mouse, and tree shrew 
(Tupaia glis), which might affect the function of the LDL receptors [33]. The human ApoE protein has Arg (encoded by UGC) instead of Cys residues associated with type 3 hyperlipidemia [12]. Moreover, there are 10 CGC codes and 20 CGG codes in the gerbil ApoE, which is greater compared to rat, mouse, human, gorilla, and baboon $[26,28]$. In addition, the deletion of a $5^{\prime}$-UTR region of the intron might influence the regulation of expression. The result suggested that the structure and function of $\mathrm{ApoE}$ in rodents are analogous to the E4 variant of human plasma ApoE [34].

ApoE can be synthesized and secreted in various mammalian tissues, including liver, brain, spleen, lung, ovary, kidney, and muscle [35]. Approximately $75 \%$ of ApoE in blood is synthesized by the liver, followed by the brain [36], and this study confirmed by immunohistochemistry that ApoE is upregulated/expressed in many organs and tissues in the gerbil and is highly expressed in the brain and kidney of gerbils with hyperlipidemia.

Mongolian gerbil is more susceptible to high fat diet and less time-consuming in forming hyperlipidemia during our many experiments or research works in many years, with similar response in increased LDL-C levels to cholesterol as human. Moreover, we found for the first time that some old individuals manifest a spontaneous hyperlipidemia in our close breeding population. It is crucially important to evaluate a new gerbil hyperlipidemia model, so that the highly expressed genes in diseased organs (such as liver) will be studied and major genes or functional SNPs will also be discovered. SNPs of the human ApoE gene show significant differences among nations or areas $[37,38]$. There is no age or sex difference in the distribution of genotype frequencies but there are differences in genotype frequency among diverse ethnicities [39]. There are few reports of SNPs in rat and mouse and only 7 of SNPs in pig [24]. SNPs within the promoter region exert a marked influence on transcription of the porcine ApoE gene [40]. However, the ApoE gene is closely associated with porcine plasma ApoE protein, LDL and cholesterol concentration, atherosclerosis, and Alzheimer's disease. Three SNPs $(97(\mathrm{C}>\mathrm{T}), 781(\mathrm{G}>\mathrm{T})$, and $1774(\mathrm{~A}>\mathrm{T}))$ mutations in the gerbil did not result in a change of the polarity of amino acids.

\section{Conclusion}

Mongolian gerbil might be a potential model for drug assessment and lipid metabolism. The apolipoprotein E (ApoE) gene includes an open reading frame of $948 \mathrm{bp}$, encoding a protein of 298 amino acids, which is analogous to human Apo E4. ApoE gene is upregulated/expressed in many organs and tissues in the gerbil and is highly expressed in the brain and kidney of gerbils with hyperlipidemia. Three new SNPs, age, and gender were significantly associated with hyperlipidemia.

\section{Conflict of Interests}

The authors declare that there is no conflict of interests regarding the publication of this paper.

\section{Authors' Contribution}

The work presented here was carried out in collaboration between all authors. Yue Huan Liu, Jiu Sheng Wu, and Ning-Ying Xu conceived and designed the experiments; Yue Huan Liu, Qiaojuan Shi, and Hong Gang Guo performed the experiments; Yue Huan Liu and Jiu Sheng Wu analyzed the data. Reagents, materials, and analysis tools were provided by Yue Huan Liu, Jiu Sheng Wu, and Hua Zhong Ying. Yue Huan Liu, Jiu Sheng Wu, and Ning-Ying Xu wrote the paper. All authors have contributed to, seen, and approved the paper.

\section{Acknowledgments}

This material is based upon work funded by Zhejiang Provincial Natural Science Foundation of China under Grant (no. Y3080126) and Science and Technology Foundation (no. 2011C37096) and sponsored by Excellent Youth Talents Grants of Zhejiang Medicine and Health (2007QN001).

\section{References}

[1] P. M. Kris-Etherton and J. Dietschy, "Design criteria for studies examining individual fatty acid effects on cardiovascular disease risk factors: human and animal studies," The American Journal of Clinical Nutrition, vol. 65, no. 5, supplement 1, pp. 1590S-1596S, 1997.

[2] H. D. Ramachandran, K. Narasimhamurthy, and P. L. Raina, "Modulation of cholesterol induced hypercholesterolemia through dietary factors in indian desert gerbils (Meriones hurrianae)," Nutrition Research, vol. 23, no. 2, pp. 245-256, 2003.

[3] P. J. Babin and G. F. Gibbons, "The evolution of plasma cholesterol: direct utility or a "spandrel" of hepatic lipid metabolism?" Progress in Lipid Research, vol. 48, no. 2, pp. 73-91, 2009.

[4] D. M. Hegsted and A. Gallagher, "Dietary fat and cholesterol and serum cholesterol in the gerbil," Journal of Lipid Research, vol. 8, no. 3, pp. 210-214, 1967.

[5] M. P. Sullivan, J. J. Cerda, F. L. Robbins, C. W. Burgin, and R. J. Beatty, "The gerbil, hamster, and guinea pig as rodent models for hyperlipidemia," Laboratory Animal Science, vol. 43, no. 6, pp. 575-578, 1993.

[6] M. T. Zhong, M. Huang, and J. Lu, "Establishment of a rapid hyperlipidemic gerbil model," Acta Laboratorium Animalis Scientia Sinica, vol. 14, no. 3, pp. 217-221, 2006.

[7] H. Z. Ying, C. H. Yu, Z. Y. Wang, B. Yu, J.-M. Zang, and Y.H. Liu, "Characterization and mechanisms of lipid metabolism in high-fat diet induced hyperlipidemia gerbil model," African Journal of Biotechnology, vol. 11, no. 97, pp. 16347-16352, 2012.

[8] H. K. Das, J. McPherson, G. A. Bruns, S. K. Karathanasis, and J. L. Breslow, "Isolation, characterization, and mapping to chromosome 19 of the human apolipoprotein E gene," The Journal of Biological Chemistry, vol. 260, no. 10, pp. 6240-6247, 1985.

[9] K. S. Meir and E. Leitersdorf, "Atherosclerosis in the apolipoprotein E-deficient mouse: a decade of progress," Arteriosclerosis, Thrombosis, and Vascular Biology, vol. 24, no. 6, pp. 1006-1014, 2004.

[10] H. Knoblauch, A. Bauerfeind, C. Krähenbühl et al., "Common haplotypes in five genes influence genetic variance of LDL and 
HDL cholesterol in the general population," Human Molecular Genetics, vol. 11, no. 12, pp. 1477-1485, 2002.

[11] S. H. Zhang, R. L. Reddick, J. A. Piedrahita, and N. Maeda, "Spontaneous hypercholesterolemia and arterial lesions in mice lacking apolipoprotein E," Science, vol. 258, no. 5081, pp. 468471, 1992.

[12] G. Utermann, U. Langenbeck, U. Beisiegel, and W. Weber, "Genetics of the apolipoprotein E system in man," The American Journal of Human Genetics, vol. 32, no. 3, pp. 339-347, 1980.

[13] J. D. Smith, A. Melian, T. Leff, and J. L. Breslow, "Expression of the human apolipoprotein $\mathrm{E}$ gene is regulated by multiple positive and negative elements," The Journal of Biological Chemistry, vol. 263 , no. 17 , pp. 8300-8308, 1988.

[14] A. S. Plump, J. D. Smith, T. Hayek et al., "Severe hypercholesterolemia and atherosclerosis in apolipoprotein E- deficient mice created by homologous recombination in ES cells," Cell, vol. 71, no. 2, pp. 343-353, 1992.

[15] Y. Gao, K. Li, S. Y. Tang, and Y. Xiao, "Study on animal models for hyperlipidemia," Journal of Hygiene Research, vol. 31, no. 2, pp. 97-99, 2002.

[16] H. Z. Ying, Y. H. Liu, B. Yu, Z.-Y. Wang, J.-N. Zang, and C.-H. $\mathrm{Yu}$, "Dietary quercetin ameliorates nonalcoholic steatohepatitis induced by a high-fat diet in gerbils," Food and Chemical Toxicology, vol. 52, pp. 53-60, 2013.

[17] N. Gaudreault, N. Kumar, V. R. Olivas et al., "Macrophagespecific apoE gene repair reduces diet-induced hyperlipidemia and atherosclerosis in hypomorphic apoe mice," PLOS ONE, vol. 7, no. 5, article e35816, 2012.

[18] W. Li, Q. J. Shi, H. G. Guo et al., "Study on the formation and mechanism of NAFLD in three different rodents," Chinese Comparative Medical, vol. 20, no. 1, pp. 76-80, 2012.

[19] D. K. Spady and J. M. Dietschy, "Interaction of dietary cholesterol and triglycerides in the regulation of hepatic low density lipoprotein transport in the hamster," The Journal of Clinical Investigation, vol. 81, no. 2, pp. 300-309, 1988.

[20] L. Chan, "Apolipoprotein B, the major protein component of triglyceride-rich and low density lipoproteins," The Journal of Biological Chemistry, vol. 267, no. 36, pp. 25621-25624, 1992.

[21] A. Pronczuk, P. Khosla, and K. C. Hayes, "Dietary myristic, palmitic, and linoleic acids modulate cholesterolemia in gerbils," The FASEB Journal, vol. 8, no. 14, pp. 1191-1200, 1994.

[22] L. Difrancesco, N. H. Mercer, and D. H. Percy, "The Mongolian gerbil as an animal model for the study of atherosclerosis," The FASEB Jounal, vol. 3, article 618, 1989.

[23] L. S. Dieter and S. Estus, "Isoform of APOE with retained intron 3; quantitation and identification of an associated single nucleotide polymorphism," Molecular Neurodegeneration, vol. 5, no. 1, article 34, 2010.

[24] J. J. Ramsoondar, E. B. Rucker, J. C. Vasquez et al., "Isolation and genetic characterization of the porcine apolipoprotein E gene," Animal Genetics, vol. 29, no. 1, pp. 43-47, 1998.

[25] D. M. Church, V. A. Schneider, T. Graves et al., "Modernizing reference genome assemblies," PLoS Biology, vol. 9, no. 7, article e1001091, 2011.

[26] R. A. Gibbs, G. M. Weinstock, M. L. Metzker et al., "Genome sequence of the Brown Norway rat yields insights into mammalian evolution," Nature, vol. 428, no. 6982, pp. 493-521, 2004.

[27] K. Lindblad-Toh, C. M. Wade, T. S. Mikkelsen et al., "Genome sequence, comparative analysis and haplotype structure of the domestic dog," Nature, vol. 438, no. 7069, pp. 803-819, 2005.
[28] A. Scally, J. Y. Dutheil, L. W. Hillier et al., "Insights into hominid evolution from the gorilla genome sequence," Nature, vol. 483, no. 7388, pp. 169-175, 2012.

[29] M. S. Boguski, N. Elshourbagy, J. M. Taylor, and J. I. Gordon, "Comparative analysis of repeated sequences in rat apolipoproteins A-I, A-IV, and E," Proceedings of the National Academy of Sciences of the United States of America, vol. 82, no. 4, pp. 992996, 1985.

[30] S. C. Williams, S. M. Bruckheimer, A. J. Lusis, R. C. LeBoeuf, and A. J. Kinniburgh, "Mouse apolipoprotein A-IV gene: nucleotide sequence and induction by a high-lipid diet," Molecular and Cellular Biology, vol. 6, no. 11, pp. 3807-3814, 1986.

[31] S. M. Fullerton, A. V. Buchanan, V. A. Sonpar et al., "The effects of scale: variation in the $A P O A_{1} / C_{3} / A_{4} / A_{5}$ gene cluster," Human Genetics, vol. 115, no. 1, pp. 36-56, 2004.

[32] S. C. Rall Jr., K. H. Weisgraber, and R. W. Mahley, "Human apolipoprotein $\mathrm{E}$ the complete amino acid sequence," The Journal of Biological Chemistry, vol. 257, no. 8, pp. 4171-4178, 1982.

[33] J. Zhang, W. W. Zeng, B. S. Chen et al., "Analysis of apolipoprotein E cDNA and protein structure of tree shrew," Chinese Jounal of Biochemistry and Molecular Biology, vol. 18, no. 1, pp. 9-13, 2002.

[34] J. W. Mclean, C. Fukazawa, and J. M. Taylor, "Rat apolipoprotein E mRNA (cloning and sequencing of double-stranded cDNA)," The Journal of Biological Chemistry, vol. 258, no. 14, pp. 89939000, 1983.

[35] M. Murakami, Y. Ushio, Y. Morino, T. Ohta, and Y. Matsukado, "Immunohistochemical localization of apolipoprotein $\mathrm{E}$ in human glial neoplasms," The Journal of Clinical Investigation, vol. 82, no. 1, pp. 177-188, 1988.

[36] C.-T. Lin, Y. F. Xu, J.-Y. Wu, and L. Chan, "Immunoreactive apolipoprotein $\mathrm{E}$ is a widely distributed cellular protein," The Journal of Clinical Investigation, vol. 78, no. 4, pp. 947-958, 1986.

[37] D. A. Nickerson, S. L. Taylor, S. M. Fullerton et al., "Sequence diversity and large-scale typing of SNPs in the human apolipoprotein E gene," Genome Research, vol. 10, no. 10, pp. $1532-1545,2000$.

[38] Q. Y. Guo, S. F. Long, P. Fan et al., "Study on the apolipoprotein E polymorphism in Chinese maonan ethnic minority," Modern Preventive Medicine, vol. 38, no. 2, pp. 324-326, 2011.

[39] X. H. Zhou, X. M. Luo, Y. Hong et al., "Association study of apolipoprotein E gene polymorphism and Alzheimer disease in Xinjiang Uygur population and Han population," Chinese Journal of Psychiatry, vol. 41, no. 4, pp. 220-224, 2008.

[40] S. Li, H. Zhang, P. Gao, Z. Chen, C. Wang, and J. Li, "A functional mutation at position -155 in porcine APOE promoter affects gene expression," BMC Genetics, vol. 12, article 40, 2011. 

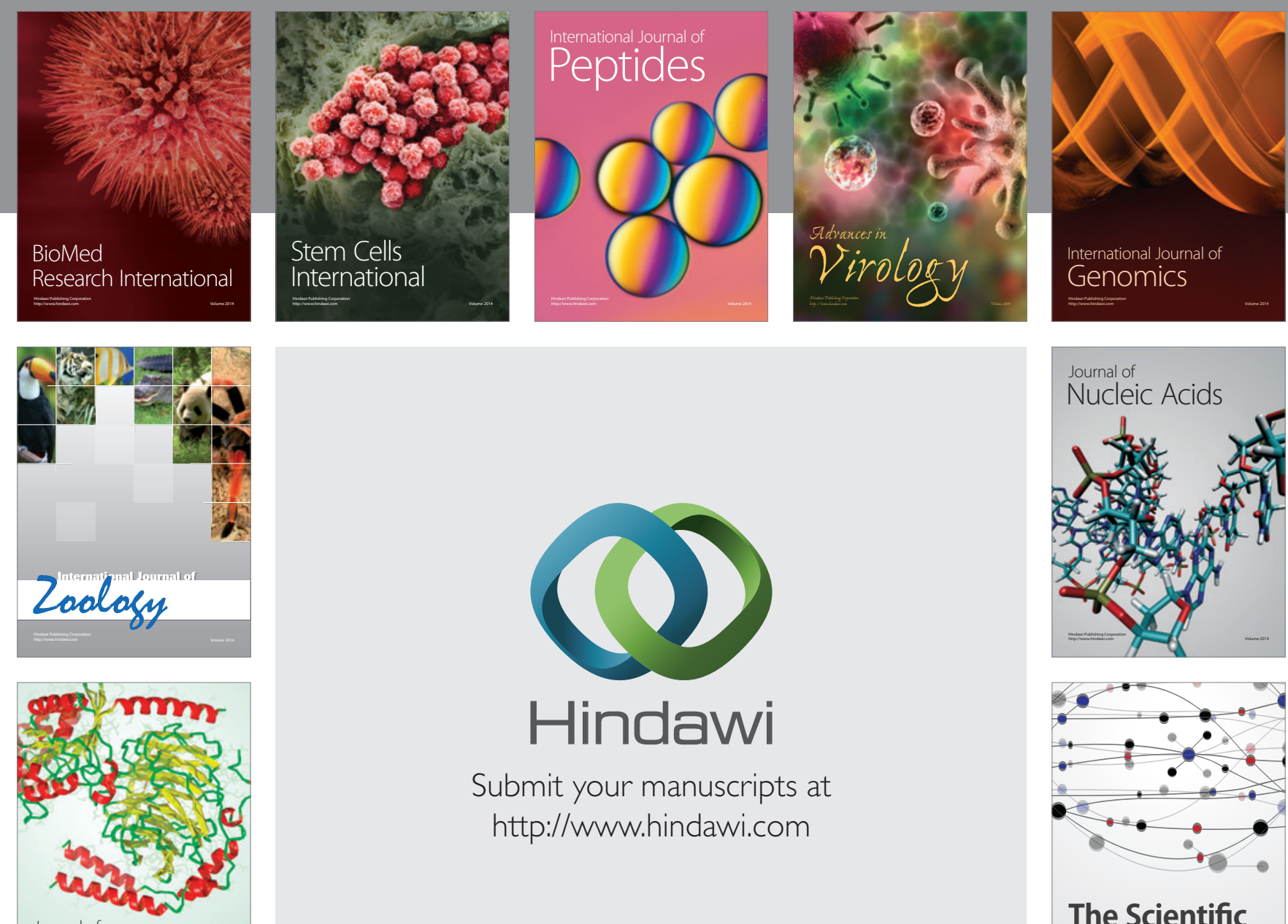

Submit your manuscripts at

http://www.hindawi.com

Journal of
Signal Transduction
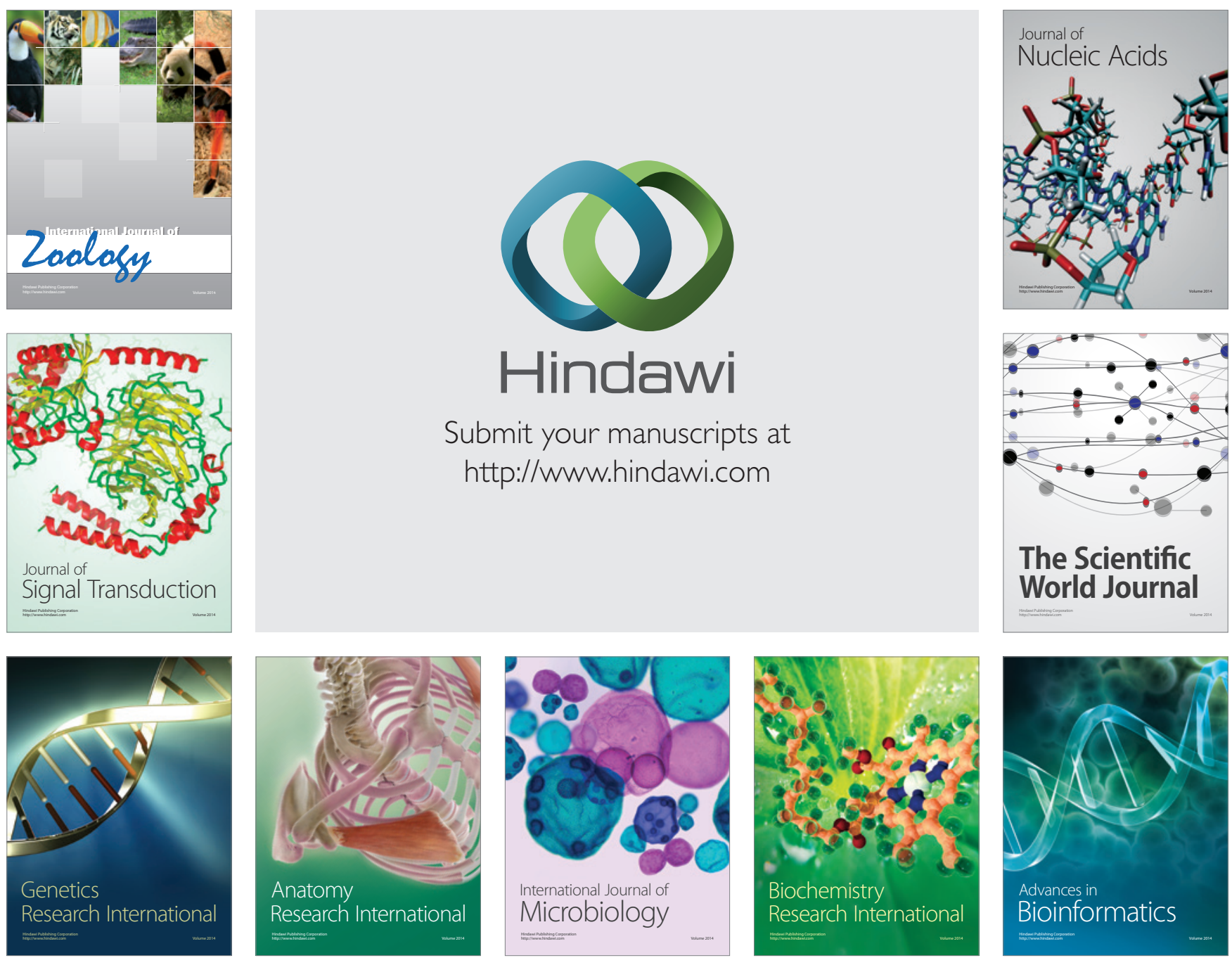

The Scientific World Journal
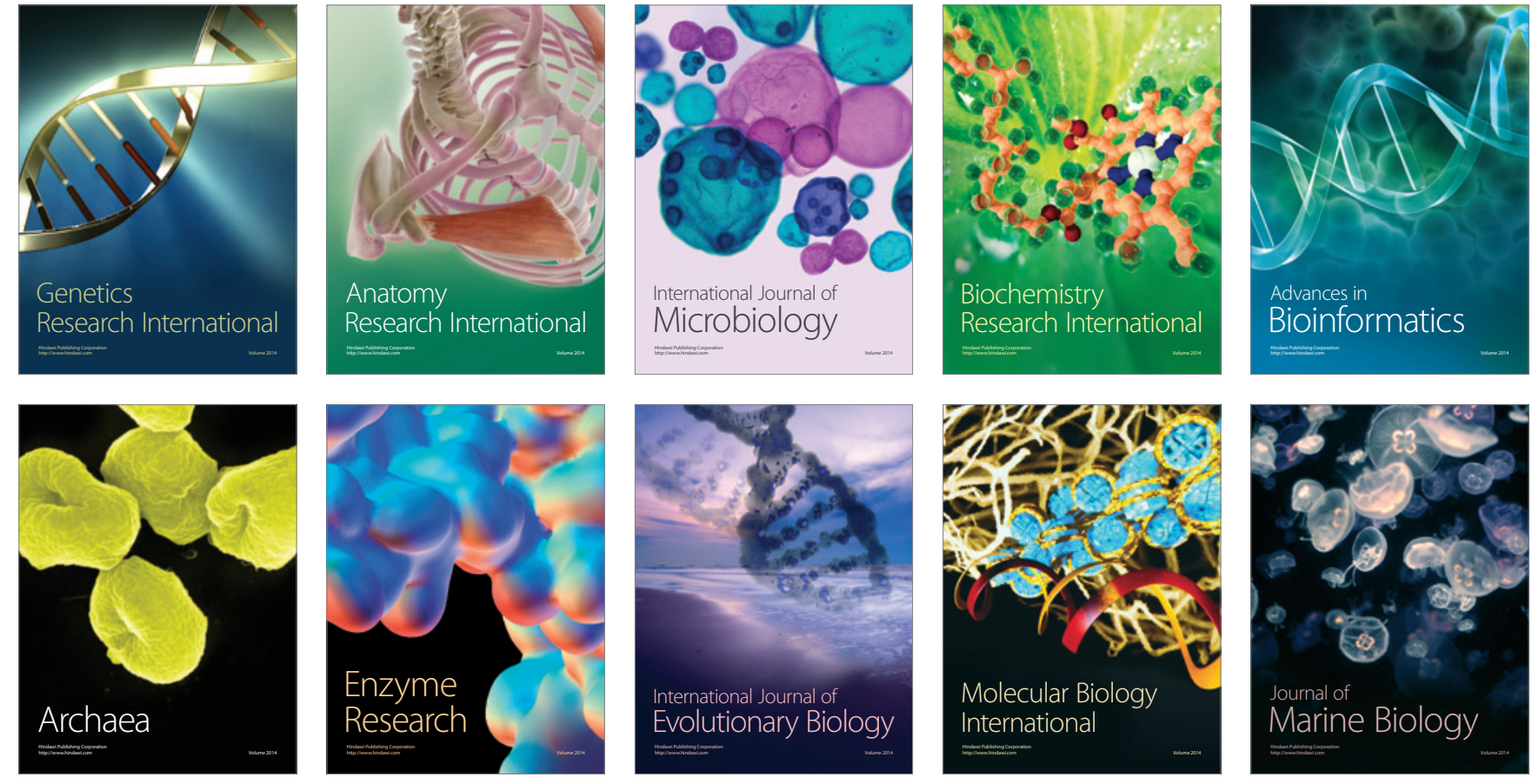